\title{
Argumentatividad: qué es y cómo medirla. El caso en el discurso de adultos mayores y jóvenes universitarios chilenos ${ }^{1}$
}

\author{
Cristián Santibáñez(1) \\ Universidad Católica de la Santísima Concepción \\ Concepción, Chile \\ José Ángel Gascón(D) \\ Universidad Católica del Maule \\ Talca, Chile
}

\section{Resumen}

En este trabajo reportamos los resultados de una investigación empírica respecto de la argumentatividad en el discurso de adultos mayores y jóvenes universitarios chilenos. Por argumentatividad se entiende la disposición a entregar o evadir la entrega de razones en un diálogo que contiene una diferencia de opinión. Desde el punto de vista teórico, este trabajo discute distintas aproximaciones al concepto de argumentatividad, como por ejemplo las provenientes de la psicología del razonamiento o la teoría de la comunicación, para finalizar con una propuesta conceptual propia. Desde el punto de vista metodológico, se utilizó una entrevista semiestructurada que contenía el uso de distintos tipos patrones argumentativos respecto de los que el entrevistado debía responder. Entre los resultados más importantes, se encontró que la edad sí es un factor particular que determina parte de la capacidad/actitud de argumentar; que el género del hablante no establece nada a priori si una persona se acerca o evita la argumentación. Finalmente, se sugieren algunas ideas para profundizar este tipo de investigaciones.

Palabras clave: argumentatividad; adultos mayores; adultos jóvenes; punto de vista; razones; revisión epistémica.

\footnotetext{
${ }^{1}$ Este trabajo forma parte de los resultados de dos investigaciones. La primera es el proyecto Fondecyt Regular No. 1170492, titulado Caracterización lingüística y cognitiva de la competencia argumentativa en adultos mayores en Chile: un estudio en las Regiones del Bio-Bio, Coquimbo y Metropolitana; y la segunda es el proyecto postdoctoral Fondecyt No. 3190149, titulado La teoría de la argumentación a la luz de la psicología del razonamiento: un análisis de las dimensiones epistémica y práctica.
} 


\begin{abstract}
Argumentativeness: What is it and how to measure it. The case in the discourse of Elderly People and Young Chilean University Students

This paper reports the results of an empirical study on argumentativeness in the discourse of Chilean elderly people and young university students. Argumentativeness means speaker's willingness to deliver or evade the use of reasons in a dialogue when there is a difference of opinion. From the theoretical point of view, different approaches to the concept of argumentativeness are reviewed, including those proposed from the psychology of reasoning or the theory of communication, in order to come up with a new and original conceptual framework. From the methodological point of view, a semi-structured interview was used. In the interview, different types of argumentative patterns were presented to which the interviewee had to respond. Among the most important results, it was found that while age is, in fact, a particular factor that partially influences a person's argumentativeness, gender is not. Finally, some ideas to further research in this area are suggested.
\end{abstract}

Key words: argumentativeness; elderly people; young people; point of view; reasons; epistemic revision.

\title{
Résumé
}

Argumentativité : qu'est-ce que c'est et comment le mesurer. Le cas dans le discours des personnes âgées et des jeunes étudiants universitaires chiliens

276 Dans cet article, nous rapportons les résultats d'une recherche empirique concernant l'argumentation dans le discours des personnes âgées chiliennes et des jeunes étudiants universitaires. Par argumentativité, nous entendons la volonté de livrer ou de se soustraire à l'utilisation de raisons dans un dialogue contenant une divergence d'opinions. Du point de vue théorique, ce travail aborde différentes approches du concept d'argumentation, telles que celles proposées par la psychologie du raisonnement ou la théorie de la communication, afin de proposer un nouveau cadre conceptuel. Du point de vue méthodologique, un entretien semi-structuré a été utilisé qui contenait l'utilisation de différents types de schémas argumentatifs auxquels la personne interrogée devait répondre. Parmi les résultats les plus importants, il a été constaté qu'en fait, l'âge est un facteur particulier qui détermine une partie de la capacité / attitude à argumenter; que le sexe du locuteur n'établit rien a priori si une personne s'approche ou évite l'argumentation. Enfin, quelques idées sont suggérées pour approfondir ce type de recherche.

Mots-clés: argumentativité ; adultes majeurs; jeunes adultes; point de vue; les raisons ; revue épistémique. 


\section{SOBRE LOS AUTORES}

\section{Cristián Santibáñez}

Investigador CIEDE, Universidad Católica de la Santísima Concepción, Ph.D. en Lingüística, trabaja en teoría de la argumentación, cognición y epistemología.

Correo electrónico: csantibanez@ucsc.cl

\section{José Ángel Gascón}

Profesor Asistente, Departamento de Filosofía, Universidad Católica del Maule; Doctor en Filosofía, trabaja en filosofía analítica, epistemología, teoría de la argumentación.

Correo electrónico: jgascon@ucm.cl

\section{CÓMO CITAR ESTE ARTÍCULO}

Santibáñez C., \& Gascón J.A. (2021). Argumentatividad: qué es y cómo medirla. El caso en el discurso de adultos mayores y jóvenes universitarios chilenos. Lenguaje, 49(2), 275-302. https://doi.org/10.25100/lenguaje.v49i2.10723. 


\section{INTRODUCCIÓN}

De acuerdo con parte de la literatura en psicología del razonamiento (Mercier, 2020; Mercier y Sperber, 2017), nuestra disposición a argumentar, a involucrarnos en conversaciones que contienen controversias, es menos sistemática y preponderante de lo que solemos creer. Desde el punto de vista de estudios empíricos de la práctica argumentativa (Hample, 2005, 2018; Hample y Anagondahalli, 2015; Hample y Dallinger, 1987; Hample et al., 2013; Hample et al., 2009), nos comunicamos argumentativamente según marcos de personalidad que fijan ciertos parámetros que determinan nuestro comportamiento argumentativo, como por ejemplo cuando nos involucramos por entretención porque poseemos una tendencia lúdica ligada a los conflictos de opinión, o cuando lo hacemos para resolver honesta y concienzudamente la diferencia; y de acuerdo con acercamientos cognitivos (Paglieri y Castelfranchi, 2010), antes que involucrarnos en diferencias de opinión, revisamos varios elementos que nos indicaría si valdría la pena hacerlo, balaceando los costos, los beneficios y las metas extradialógicas en torno al conflicto de opinión que potencialmente podríamos enfrentar.

Estas caracterizaciones de la disposición a argumentar pueden ser capturadas, conceptualmente, en una sola noción que puede llamarse argumentatividad. Este trabajo intenta dar algunos puntos de inicio en lo relativo a cómo definir y entender el concepto. Para este efecto, también se reporta aquí un estudio piloto que dispuso de una metodología para analizarla. En otras palabras, este trabajo ofrece una discusión para discernir qué es la argumentatividad y cómo podemos medirla. Nuestro objetivo fue analizar exploratoriamente el comportamiento argumentativo de dos grupos de hablantes (adultos mayores y adultos jóvenes universitarios, respectivamente), considerando específicamente el comportamiento lingüístico manifestado por las personas ante un estímulo argumentativo contenido en una entrevista semiestructurada.

El artículo avanza siguiendo el siguiente orden: en la sección Argumentatividad: Qué es y cómo medirla, ofrecemos un panorama de lo que puede considerarse componentes de un concepto de argumentatividad, componentes que provienen de reflexiones en áreas disciplinarias distintas pero emparentadas, culminando esta sección con una propuesta teórica; en la sección Metodología, detallamos los aspectos metodológicos del estudio empírico que realizamos para observar el rendimiento de la propuesta teórica diseñada, en este caso analizando la argumentatividad en adultos mayores chilenos; en la sección Hallazgos y discusión, presentamos los hallazgos principales y su discusión; y ya en las conclusiones, indicamos algunas líneas de desarrollo futuro que podrían fortalecer la conceptualización y el estudio empírico de lo que denominamos argumentatividad. 


\section{Argumentatividad: qué es y cómo medirla}

En esta sección ofrecemos una revisión de la literatura sobre el tema que abordamos y que entrega un contexto conceptual elemental que ayuda a estructurar el término de argumentatividad. Nuestra revisión comienza con algunos elementos provenientes de la psicología del razonamiento; luego continúa con la teoría económica de la argumentatividad; y finalmente revisamos los aportes de la teoría de los marcos argumentativos. En la subsección de la Propuesta conceptual, ofrecemos nuestra definición de argumentatividad, integrando algunos de los elementos previamente discutidos y, al mismo tiempo, entregando otros nuevos para dar forma conceptual al término que posteriormente sometemos a un desafío empírico analizando la argumentatividad demostrada por adultos mayores chilenos.

\section{Psicología del razonamiento: argumentatividad conservadora}

En una publicación reciente (Mercier, 2020), se ha indicado que poseemos una tendencia, aunque esporádica y puntual, a obstinadamente mantener nuestras creencias y opiniones, incluso resistiendo evidencia objetiva, pública y confiable. Conocido en inglés como backfire effect, que muestra la disposición ciega de las personas a perseverar en sus creencias, esta actitud motiva el comportamiento de defender la misma posición a pesar de la contundente contraevidencia, utilizando los mismos argumentos (razones) con los que iniciamos la defensa del punto de vista. Como se indicó, aunque esta tendencia es puntual y practicada, en raras ocasiones nos ayuda a entender que la disposición a argumentar está limitada por ciertos parámetros psicológicos y motivacionales específicos, tales como el sesgo de la confirmación, que es la tendencia a buscar, interpretar y evaluar información de manera que apoye nuestros propios puntos de vista. Mercier (2020) indica evidencia que, si se nos presenta información confiable y sólida, tendemos a cambiar en algo nuestra creencia inicial hacia la dirección a la que la nueva información orienta, dependiendo también fuertemente de la fuente que utiliza la información en su argumento. Un ejemplo real de esta actitud se observa en el comportamiento del expresidente estadounidense George W. Bush y su gobierno que para justificar la invasión a Irak argumentaron la existencia de armas nucleares escondidas en aquel país, y esta creencia persistió por años con muy pocos cambios a pesar de la contundente evidencia de lo contrario, vale decir, de la inexistencia de armas de destrucción masiva (Mercier, 2020).

Estas ideas de Mercier, que revisa una extensa bibliografía principalmente en el ámbito de la psicología del razonamiento, apoyarían la opinión de que tenderíamos a morigerar nuestras participaciones argumentativas, ser proclives al intercambio de creencias, particularmente porque existiría un tipo de defensa tendiente a preservar la consistencia de nuestra red de creencias incluso cuando nos enfrentamos a evidencia contundente. Se podría concluir, entonces, que desde la perspectiva que defienden varios autores (Mercier, 2011a, 2011b, 2011c, 2011d, 2012; Mercier y Landemore, 2012; Mercier y Sperber, 2009; Sperber, 2001; Sperber y Mercier, 2012; Sperber et al., 2010), 
la argumentatividad de las personas, definida por ahora de forma simple como la tendencia a involucrarse en intercambios controversiales, estaría limitada por una estructura cognitiva orientada hacia conservación de las creencias. El sesgo de confirmación sería un poderoso heurístico que nos confina a argumentar solo hasta allí donde podemos justificar algo, sin hacer el esfuerzo por encontrar el argumento óptimo.

Para muchos efectos de la vida diaria, los humanos tendemos a tomar decisiones que incorporan el balance de escenarios y razones de forma intuitiva, ya que la mente estaría diseñada dualmente, así como se ha esforzado una gran cantidad de autores por demostrarlo (Evans, 2010; Evans y Frankish, 2009; Kahneman, 2011; Manktelow et al., 2011; Stanovich, 2010; Stanovich y Toplak, 2012). Este diseño complotaría con la imagen de una capacidad siempre dispuesta al servicio de justificar adecuadamente nuestros puntos de vista $y$, con esta posibilidad, participar sin miramientos en intercambios argumentativos. Dicho de otra forma, este diseño cognitivo estructural complotaría contra una argumentatividad inherente. Por el contrario, solo cuando hacemos uso del sistema reflexivo, nuestra potencial capacidad argumentativa estaría en marcha. Según Dessalles (2000/2007), solo estaríamos en el modo argumentativo cuando tratamos de encontrar una solución a un conflicto cognitivo, y un conflicto cognitivo se da cuando las creencias y deseos que constituyen el conflicto tienen cierto grado de intensidad contradictoria.

Teoría económica de la argumentatividad

Una posición similar, pero desde un ángulo teórico en algo diferente, es la que sostienen Paglieri y Castelfranchi (2010) cuando indican que habría cierta reticencia a argumentar en consideración a los costos, beneficios y obstáculos o peligros que el intercambio argumentativo pudiera generar. Esta perspectiva es similar a la anterior proveniente de la psicología del razonamiento al concebir que habría consideraciones funcionales y estratégicas del agente que, automática o cuasi reflexivamente, toma en cuenta antes de insertarse en un intercambio de opiniones. Para Paglieri y Castelfranchi (2010) no incursionaríamos en disputas cuando prevemos resultados negativos. Como se ha indicado en otros lugares (Santibáñez, 2014), estos autores conciben que argumentar es una actividad demandante y puede generar resultados adversos a los que uno pudiera planear.

Estos cálculos funcionarían como filtros para decidir si argumentar o no, y en la práctica pueden desmotivar una gran cantidad de impulsos por intercambiar opiniones. De modo que la tendencia potencial a involucrarse en actividades argumentativas se limita ostensiblemente. Paglieri y Castelfranchi (2010) defienden una posición sobre la argumentación que puede llamarse como instrumentalista, vale decir, el cálculo que harían los agentes para involucrarse en la actividad argumentativa está en relación con distintos tipos de metas dialógicas y extra dialógicas que pudieran estar convergiendo para ese agente en un contexto particular: el agente balanceará tema de disputa, participantes del diálogo, participantes 
diferidos, metas del diálogo, metas diferidas, etc. Como todo instrumentalismo, éste también está guiado por la búsqueda de la utilidad. Y la utilidad para un agente en la actividad argumentativa estaría dada, para estos autores, en directa relación tanto con la dimensión próxima del intercambio conflictivo (convencer respecto de una creencia, lograr que el oyente oriente su acción de acuerdo con una creencia esgrimida, etc.), como con la dimensión ulterior (relativa a efectos colaterales positivos, tales como: reputación, confiabilidad, credibilidad). En su reverso, la utilidad adversa, o negativa, está vinculada directamente a los costos y peligros. Si los costos involucran una oportunidad perdida, vale decir, un curso de acción alternativo que con el tiempo se observa que fue más beneficioso, la decisión original se avalúa como defectuosa, o costosa. De modo que están los costos directos y los costos diferidos. Los peligros, en cambio, dicen relación con efectos extendidos, o colaterales, que se producen, por ejemplo, cuando perdemos cercanía con una persona por efecto de una conversación que contuvo una clara diferencia de opinión y respecto de la que las partes no encontraron solución. Para que el acto de argumentar sea netamente beneficioso, la utilidad esperada siempre debe ser mayor a los costos y peligros. Pero el problema es que muchas veces ambos, costos y peligros, son difíciles de imaginar, sopesar o advertir. De modo que, por esto, habría una reticencia a argumentar de forma constante o sistemática, lo que podría explicar, por ejemplo, que a pesar de los esfuerzos de gobiernos para que la gente participe de discusiones ciudadanas y sociales, las personas no lo hagan. Costos adheridos prototípicos a la actividad de argumentar son los de la exposición social, consumo de energía, tiempo e incerteza del resultado en términos del rendimiento de adoptar una nueva creencia u optar por un nuevo curso de acción. Esta visión económica, la de proyectar la intersección de las curvas de beneficios y costos, tiende a representar la argumentación, sostenida en el tiempo, como una actividad que, en algún punto, desgastaría al argumentador, ya que aumenta exponencialmente la exposición social y hace disminuir la energía y el tiempo disponible. Bajo esta perspectiva, una predicción evidente es que la argumentatividad, esa tendencia potencial de los individuos a involucrarse en un conflicto de opinión se reduce con el paso del tiempo irremediablemente.

\section{Marcos argumentativos}

El concepto de argumentatividad está explícitamente utilizado en el acercamiento de Hample (2005) que podemos denominar teoría de los marcos argumentativos. Esta teoría, de acuerdo con su principal cultor (Hample, 2005, 2018), recoge instrumentos provenientes mayoritariamente de la psicología social para medir comportamientos y expectativas en el contexto de la comunicación cara a cara con perfil controversial. Como se ha indicado en otras publicaciones (Santibáñez y Hample, 2015), un primer conjunto de medidas busca caracterizar las orientaciones motivacionales básicas para la práctica de la argumentación interpersonal (Rancer y Avtgis, 2006), que es, precisamente, la tendencia a argumentar, o argumentatividad (Infante y Rancer, 1996), pero también la agresividad verbal (Infante y Wigley, 1986). Ambas variables, 
argumentatividad y agresividad verbal, son medidas a través de dos subescalas, una rastrea los impulsos de acercamiento, y la otra los impulsos a evadir. En el caso de la argumentatividad, que Hample (2005) define como la predisposición a atacar el punto de vista, las pruebas o razones de otra persona, la subescala de aproximación/evasión evalúa si el respondiente se inclina a participar en ese tipo de comunicación o si prefiere evitarlo. Para el caso de la agresividad verbal, que se define como la predisposición a atacar el carácter de los otros interactuantes, su identidad, historia personal, u otras características de la persona, las subescalas de aproximación/evasión miden la inclinación a ser antisocial (hostil, desagradable) o prosocial (educado, cuidadoso en lo que se dice).

Un segundo gran grupo de instrumentos de medición para el estudio empírico de la interacción comunicativa cara a cara que incluye la actividad de dar razones en esta teoría describe los marcos argumentativos de las personas. Este conjunto de instrumentos se desarrolló para responder a la pregunta: ¿Qué cree que hace la gente común cuando argumenta? Con el concepto de marcos argumentativos, Hample (2005) intenta capturar las expectativas y orientaciones relativas a la argumentación interpersonal. Este autor distinguió tres categorías generales de marcos argumentativos. La primera es el egocentrismo, que se relaciona con el uso de razones personales en la argumentación. Dentro del egocentrismo, el hablante quizás busque argumentar por utilidad (obtención de algún beneficio), exhibir identidad (para mostrar algunas características personales), expresar dominancia (el hablante intenta 282 demostrar que es superior a su adversario en algunos aspectos) o jugar (argumentar por entretenimiento). Estos objetivos enfatizan la personalidad y caracterizan al otro argumentador como un medio para conseguir metas propias. La segunda categoría del conjunto que compone los marcos argumentativos se relaciona con la orientación hacia el otro, vale decir, al interlocutor se le toma en cuenta de manera más auténtica. Los instrumentos utilizados en esta categoría buscan rastrear si el hablante tiene en la actividad argumentativa una tendencia impulsiva (el hablante impulsivo dice lo que piensa, mientras que quien no lo es adapta sus expresiones a las necesidades de la otra persona); una tendencia cooperativa (siendo su opuesto la competencia en la que se toma en cuenta al otro pero de forma manipuladora), o una tendencia hacia la civilidad (que caracteriza a un hablante como cortés y un carácter constructivo). La última categoría de los marcos argumentativos se refiere al conjunto de medidas que rastrean los niveles de reflexividad de un hablante, a partir de lo que Hample (2005) denomina "contraste profesional". Este conjunto de medidas permite, por ejemplo, demostrar que mientras los teóricos de la argumentación piensan que argumentar es una alternativa a la violencia, la gente común considera que la argumentación puede conducir a la violencia. Una puntuación alta en este instrumento significa que el argumentador cotidiano converge con los teóricos de la argumentación.

Una nota historiográfica de la noción de marcos argumentativos que Hample (2005) usa ayuda a cerrar esta sección. Hample se basa en la idea de marcos que Goffman $(1959,1967,1969)$ planteó. Se debe recordar que para Goffman toda situación tiene un strip propio y natural, esto es, una secuencia de acciones que contienen ciertas 
claves que ayudan a los interactuantes a participar adecuadamente en un contexto comunicativo determinado, siendo algunas altamente ritualizadas. En un contexto de argumentación interpersonal, el intercambio de razones cara a cara sería su marco más natural, el que puede ser, a su vez, específicamente determinado como juego, dominancia, o de proyección de identidad, según las tendencias de quienes lo practiquen.

\section{Propuesta conceptual}

Infante y Rancer (1982) concibieron la argumentatividad como un rasgo propio de las personas, como una disposición a argumentar. Definieron la argumentatividad como una predisposición a defender puntos de vista sobre cuestiones controvertidas y a refutar las posturas de otras personas sobre esas cuestiones. Conceptualizaron la argumentatividad en términos de dos dimensiones opuestas: la tendencia a aproximarse a discusiones argumentativas y la tendencia a evitarlas. Naturalmente, la primera indica una mayor argumentatividad, mientras que la segunda reduce la puntuación de argumentatividad. Una persona con una alta argumentatividad tendrá una alta tendencia a la aproximación y una baja tendencia a la evitación. Alguien con baja argumentatividad tendrá una baja tendencia a la aproximación y una alta tendencia a la evitación. Y, si la argumentatividad es moderada, entonces ambas dimensiones serán altas o bajas al mismo tiempo. Posiblemente las personas con argumentatividad moderada sean más susceptibles a factores contextuales.

Para medir la argumentatividad, Infante y Rancer (1982) elaboraron un cuestionario de 20 preguntas en el que los individuos deben reportar sus inclinaciones y sus sentimientos hacia acciones argumentativas. Cada una de las preguntas consiste en un enunciado en el que los individuos deben indicar, en una escala de 1 a 5 , en qué medida se identifican con él. La mitad de los enunciados mide la tendencia a aproximarse a discusiones $(2,4,7,9,11,13,15,17,18,20)$ y la otra mitad mide la tendencia a evitarlas $(1,3,5,6,8,10,12,14,16,19)$. Es importante observar los tipos de preguntas para entender cómo se va construyendo la escala de argumentatividad desde el punto de vista de estos autores. Las preguntas son:

1. Durante una discusión, me preocupa que la persona con la que estoy discutiendo se forme una impresión negativa de mí.

2. Argumentar sobre cuestiones controvertidas mejora mi inteligencia.

3. Disfruto evitando discusiones.

4. Soy enérgico y entusiasta cuando argumento.

5. Cuando termino una discusión me prometo a mí mismo que no me meteré en otra.

6. Argumentar con una persona crea más problemas para mí de los que resuelve.

7. Siento una sensación buena y placentera cuando gano un punto en una discusión.

8. Cuando termino de discutir con alguien me siento nervioso y molesto. 
9. Disfruto de una buena discusión sobre alguna cuestión controvertida.

10. Siento una sensación desagradable cuando me doy cuenta de que estoy a punto de meterme en una discusión.

11. Disfruto defendiendo mi punto de vista sobre alguna cuestión.

12. Me alegro cuando evito que ocurra una discusión.

13. No me gusta perder la oportunidad de argumentar una cuestión controvertida.

14. Prefiero estar con personas que rara vez están en desacuerdo conmigo.

15. Considero una discusión como un reto intelectual excitante.

16. Me veo incapaz de pensar en observaciones eficaces durante una discusión.

17. Me siento renovado y satisfecho después de una discusión sobre una cuestión controvertida.

18. Tengo una habilidad para ser bueno en las discusiones.

19. Intento evitar meterme en discusiones.

20. Siento entusiasmo cuando veo que una conversación en la que participo está conduciendo a una discusión. (Infante y Rancer, 1982, p. 76).

Naturalmente, uno de los propósitos de la conceptualización de la argumentatividad es poder hacer predicciones sobre el comportamiento de las personas en situaciones argumentativas. La dificultad de esto reside en el hecho de que la argumentatividad es solo uno de los factores que intervienen en la decisión de participar en una discusión; diversos aspectos de la situación interactúan con la 284 disposición del argumentador para determinar esa decisión (Hample, 2005). No obstante, la validez y la fiabilidad de esta escala de argumentatividad han sido comprobadas y se ha estudiado la interacción entre este rasgo del carácter y factores contextuales (Infante y Rancer, 1996). Por ejemplo, hay indicios de que las personas con una alta argumentatividad argumentan más sobre ciertos temas (cuestiones sociales o políticas, el comportamiento personal o de otros y cuestiones éticas), mientras que, cuando se trata de otros temas (deportes, entretenimiento, cuestiones religiosas), "las personas con alta argumentatividad argumentan tanto como las que tienen una baja argumentatividad" (Infante y Rancer, 1996, p. 331). Asimismo, el nivel de argumentatividad de todas las personas involucradas en una discusión determina la motivación para argumentar de cada una (Rancer e Infante, 1985).

En el estudio que se reporta en el presente artículo, uno de los objetivos fue analizar el comportamiento argumentativo de los participantes. Para ello, en lugar de basarnos en el cuestionario de auto-reporte propuesto por Infante y Rancer, nuestro análisis de la argumentatividad se apoyó en el comportamiento manifestado por las personas ante un estímulo argumentativo. Aquí, por tanto, la argumentatividad no se conceptualiza como un rasgo del carácter, sino como un tipo de comportamiento lingüístico. Hasta donde sabemos, no hay estudios previos de la manifestación de la argumentatividad en el sentido pretendido aquí, de modo que las categorías de análisis fueron elaboradas por nosotros sobre la base de los datos obtenidos en el presente estudio. 
El punto de partida, no obstante, fue la definición de la argumentatividad de Infante y Rancer (1982) como una predisposición a defender puntos de vista sobre cuestiones controvertidas y a refutar las posturas de otras personas sobre esas cuestiones. Como se detallará en la Metodología, se estudió el comportamiento de los individuos por medio de una entrevista personal. Las respuestas que ofrecieron los entrevistados fueron agrupadas en diferentes categorías, y cada una de las categorías fue ordenada de mayor a menor argumentatividad de acuerdo con las siguientes dimensiones de evaluación:

- Esfuerzo comunicativo: Esta es la dimensión menos exigente, que distingue a los entrevistados que intentaron ofrecer alguna respuesta (del tipo que fuera) de los que no hicieron ese esfuerzo (por ejemplo, diciendo "no sé"). Quien intenta responder es más argumentativo que quien no lo intenta.

- Punto de vista: Se puede distinguir entre los entrevistados que no lograron formular un punto de vista claro, que fueron considerados menos argumentativos, y los que presentaron un punto de vista y por tanto demostraron ser más argumentativos.

- Razones: Se pueden considerar el elemento central del comportamiento argumentativo. A diferencia de las demás dimensiones, no encontramos aquí únicamente dos posibilidades, sino que se identificaron hasta cinco en las entrevistas. Se enumeran a continuación de menor a mayor argumentatividad. En primer lugar, se consideró que las respuestas menos argumentativas eran las que ofrecían comentarios irrelevantes en lugar de razones, pues eso parece manifestar una inconsciencia por parte del entrevistado de que se encuentra en un contexto argumentativo. En segundo lugar, está la ausencia de razones, que indica que el entrevistado no logró manifestar un comportamiento argumentativo, aunque pudiera ser consciente de que el contexto lo requería. En tercer lugar, algunos entrevistados se limitaron a repetir su punto de vista, quizá creyendo que estaban aportando razones. En cuarto lugar, está la presentación de razones explicativas en lugar de argumentativas, esto es, en lugar de ofrecer indicios o pruebas de que el punto de vista es correcto, dieron una explicación que asume la corrección del punto de vista. Y, por último, en el nivel más alto de argumentatividad, encontramos la presentación de razones argumentativamente pertinentes.

- Contraargumentación: Ante las preguntas que enfrentaban a los entrevistados con una contraargumentación, algunas respuestas no tuvieron en cuenta dicha contraargumentación (por ejemplo, ni dudan de su punto de vista ni intentan refutar la contraargumentación), lo que indica una menor argumentatividad. Otras respuestas, en cambio, manifestaban un reconocimiento de la contraargumentación, y por ello se consideraron más argumentativas.

- Convicción: Ante las preguntas contraargumentativas, algunas respuestas manifestaron dudas sobre el propio punto de vista y otras no. Se consideraron más argumentativas aquellas que, habiendo reconocido la 
contraargumentación, no dudaron, sino que persistieron en defender el punto de vista. Aunque el reconocimiento de la posibilidad de estar equivocado pueda considerarse una virtud, la definición de argumentatividad de Infante y Rancer (1982) implica que la defensa con convicción de un punto de vista refleja una mayor argumentatividad.

- Explicitud: Mientras que las anteriores dimensiones pueden inferirse en mayor o menor medida de la definición de Infante y Rancer (1982), esta es una nueva consideración, que se tuvo en cuenta tras valorar todas las demás dimensiones. Ante la contraargumentación, entre los entrevistados que persistieron en su punto de vista, algunos afirmaron explícitamente que ellos no estaban equivocados y otros persistieron en su argumentación sin hacer tal afirmación explícita. Se consideró que los primeros eran más argumentativos.

Estas dimensiones de evaluación, tomadas conjuntamente, sirven para conceptualizar la argumentatividad como una manifestación comportamental de los hablantes en un contexto argumentativo. En la sección Metodología se mostrará de qué modo estas dimensiones nos permiten valorar numéricamente las respuestas de los entrevistados.

\section{Metodología}

\section{Alcance de la investigación}

Nuestro diseño investigativo tiene un alcance exploratorio y descriptivo, utiliza un instrumento de carácter cualitativo (una entrevista estructurada) y procede con un análisis argumentativo de las respuestas al cuestionario, y tres de las dimensiones de nuestra escala (a saber, Esfuerzo comunicativo, Convicción y Explicitud) se construyeron a partir de un diseño teórico fundamentado, vale decir, a partir del descubrimiento de tendencias o patrones en las respuestas mismas. Al tener un alcance exploratorio y descriptivo, el trabajo no avanza una hipótesis específica.

\section{Participantes y muestra}

La muestra fue de carácter no probabilística. Estuvo compuesta por adultos mayores (sobre 65 años) de tres regiones de Chile: de Bío-Bío, Coquimbo y Metropolitana, y por jóvenes universitarios (entre 18 y 26 años) de dos regiones: Coquimbo y Metropolitana. Se accedió a los adultos mayores utilizando los registros de unidades de salud, así como del Servicio Nacional del Adulto Mayor del Gobierno de Chile (SENAMA), entre otras fuentes de información. A su vez, se accedió a los jóvenes universitarios que circulaban por los campus universitarios.

En el caso de los adultos mayores, se pudo registrar 4 variables independientes básicas y que forman parte de los análisis más adelante entregados: 1) Edad, la que se ordenó en dos tramos etarios: de 65 a 74 años, y sobre 75 años; 2) el nivel socioeconómico, exceptuando la participación de población de extrema 
vulnerabilidad; 3) nivel educativo; y 4) género de los participantes. Ambas muestras (adultos mayores y jóvenes universitarios) firmaron voluntariamente un consentimiento informado para participar.

\section{Instrumento de recolección de datos}

Se utilizó una entrevista estructurada cuya duración en su aplicación para el caso de los adultos mayores (AM) fue de alrededor de 20 minutos y, en el caso de los jóvenes (JO), 23 minutos en promedio. La entrevista midió específicamente algunas capacidades argumentativas que se detallan en la Tabla 1: 
Tabla 1. Instrumento Entrevista Estructurada

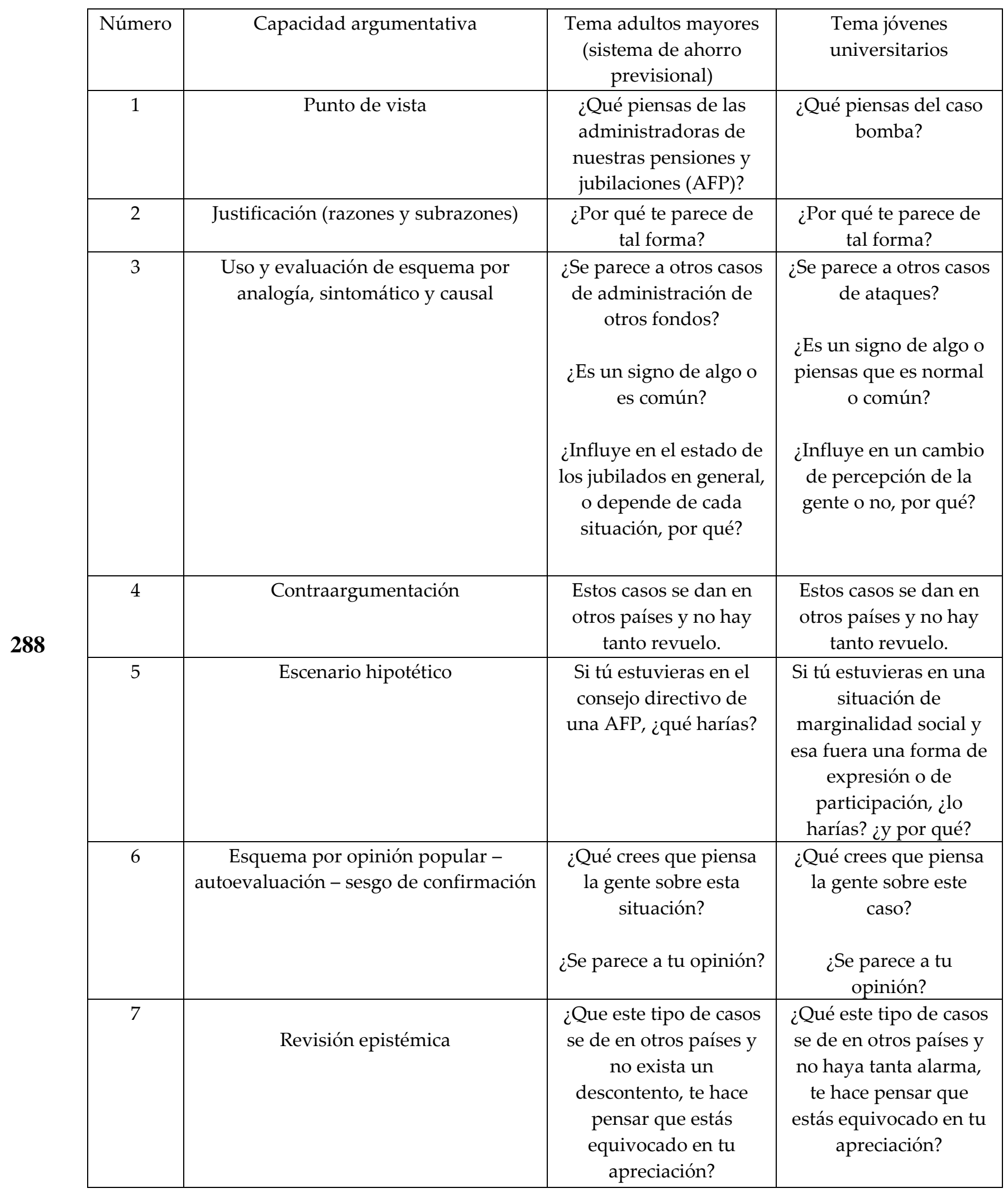

Como se observa, los temas para ambos grupos de participantes fueron distintos. La diferencia temática solo radica en conversar sobre un asunto respecto del que el respondiente tiene información y tiene cierta cercanía vital. En el caso de los 
adultos mayores el tema del sistema de ahorro previsional es de importancia fundamental y, del mismo modo, el tema de un ataque con bomba en la vía pública perpetrado por jóvenes tuvo una resonancia importante en este grupo etario. Se escogió el tema del ahorro previsional para los adultos mayores ya que en Chile se ha observado una discusión social y política larga y sistemática respecto de cambiar el sistema que hoy tiene como gran administrador de los fondos empresas privadas, siendo el caso que parte mayoritaria de la ciudadanía considera que sería óptimo transitar hacia un sistema de reparto solidario. El caso de un ataque con bomba a una estación del metro de Santiago de Chile fue, de acuerdo con la investigación, perpetrado por estudiantes universitarios pertenecientes a grupos políticos anárquicos que se movilizan para reclamar en contra de un país muy injusto. En esta investigación se está consciente de que la cercanía o importancia de los temas para los/las entrevistados/as puede generar un sesgo en los resultados, pero el riesgo de ofrecer un tema lejano o de poca relevancia podía generar el problema de la producción de poco discurso en los/as entrevistados/as.

Por otra parte, se puede observar que la segunda columna representa el comportamiento argumentativo medido: la capacidad de expresar punto de vista; la capacidad de expresar razones; la capacidad de distinguir tipos de esquemas argumentativos y responder consecuentemente; la capacidad de enfrentarse a una contraargumentación; la capacidad de situarse en un escenario hipotético y producir razones en ese escenario; la capacidad de distinguir a la apelación a la opinión popular y rastrear si el hablante tiende a confirmar su punto de vista; y, finalmente, la capacidad de revisar su punto de vista.

En este estudio hemos analizado cuatro de todas las competencias específicas medidas, a saber, la capacidad de producir punto de vista, la de expresar razones para el punto de vista, la de contraargumentar y la de revisión epistémica. El análisis, por tanto, se ha centrado en las preguntas 1, 2, 4 y 7, ya que las preguntas 3,5 y 6 contenían variables analíticas de mayor complejidad relativas al uso de esquemas argumentativos y escenarios hipotéticos.

\section{Herramientas de análisis}

Una vez obtenidas las respuestas de los entrevistados a las preguntas, un primer análisis sirvió para hallar elementos comunes en muchas de ellas, de modo que fueron agrupadas en diferentes categorías. Las categorías, por tanto, emergieron a partir de un estudio detallado de las respuestas de los entrevistados (en lugar de ser propuestas a priori como hipótesis) sobre la base de las dimensiones de evaluación de la argumentatividad propuestas en nuestra Propuesta conceptual. Como ya se explicó en esa sección, aquí proponemos tales dimensiones de evaluación como manifestaciones de argumentatividad de acuerdo con la concepción de Infante y Rancer (1982). Las siguientes categorías nos permitieron agrupar las respuestas de los entrevistados usando las dimensiones de evaluación de la argumentatividad como criterios. 
A continuación, se indican cuáles fueron las categorías que se hallaron para cada pregunta:

Pregunta 1:

- Presenta un punto de vista con razones.

- Presenta un punto de vista sin razones.

- No presenta un punto de vista bien definido.

Pregunta 2:

- Ofrece razones pertinentes.

- Ofrece una explicación en lugar de una justificación de su punto de vista.

- Repite su punto de vista sin ofrecer razones.

- Hace comentarios irrelevantes.

- No sabe.

Pregunta 4:

- No acepta la contraargumentación.

- Ofrece razones que rebaten la contraargumentación.

- Muestra dudas sobre su punto de vista.

- Ofrece más razones a favor de su punto de vista.

- Responde con comentarios evasivos.

- No sabe.

Pregunta 7:

- Afirma que no puede estar equivocado y aporta razones.

- No dice explícitamente que puede estar equivocado y ofrece razones.

- Reconoce que puede estar equivocado.

- Afirma que no puede estar equivocado sin aportar razones.

- Responde con comentarios evasivos.

- No sabe.

Cada una de estas categorías puede evaluarse de acuerdo con las diferentes dimensiones de argumentatividad que se discutió en nuestra Propuesta conceptual: esfuerzo comunicativo, punto de vista, razones, contraargumentación, convicción y explicitud. Naturalmente, solo se aplicaron tales dimensiones cuando resultó pertinente; en la pregunta 1, por ejemplo, no es pertinente la dimensión de contraargumentación. La evaluación se llevó a cabo por medio de unas tablas en las que: (1) cada dimensión de evaluación sirve para distinguir unas categorías de otras, y (2) las categorías quedan ordenadas de mayor a menor argumentatividad. De este modo, se pudo asignar una puntuación de argumentatividad a cada categoría, empezando por la parte inferior de la tabla y aumentando a medida que se asciende en la tabla.

La tabla para la pregunta 1 (Tabla 2), en la que las dimensiones de evaluación pertinentes son únicamente punto de vista y razones, es: 
Tabla 2. Dimensiones pregunta 1

\begin{tabular}{|l|l|l|}
\hline \multicolumn{2}{|c|}{ Dimensiones de evaluación } & \multicolumn{1}{c|}{ Tipo de respuesta } \\
\hline Tiene punto de vista. & Da razones. & $\begin{array}{l}\text { Presenta un punto de vista con razones. } \\
(2 \text { puntos. })\end{array}$ \\
\cline { 2 - 3 } & No da razones. & $\begin{array}{l}\text { Presenta un punto de vista sin razones. } \\
(1 \text { punto. })\end{array}$ \\
\hline No tiene punto de vista. & $\begin{array}{l}\text { No presenta un punto de vista bien } \\
\text { definido. } \\
(0 \text { puntos. })\end{array}$ \\
\hline
\end{tabular}

En la pregunta 2 (Tabla 3) es posible incorporar la dimensión de evaluación esfuerzo comunicativo, ya que nos encontramos con entrevistados que ni siquiera intentaron responder. Además, en esta pregunta el nivel de argumentatividad está determinado por la dimensión razones:

Tabla 3. Dimensiones pregunta 2

\begin{tabular}{|l|l|l|}
\hline \multicolumn{2}{|c|}{ Dimensiones de evaluación } & \multicolumn{1}{c|}{ Tipo de respuesta } \\
\hline $\begin{array}{l}\text { Intenta } \\
\text { responder. }\end{array}$ & $\begin{array}{l}\text { Da razones argumentativamente } \\
\text { pertinentes. }\end{array}$ & $\begin{array}{l}\text { Ofrece razones pertinentes. } \\
(4 \text { puntos. })\end{array}$ \\
\cline { 2 - 3 } & Explica en lugar de argumentar. & $\begin{array}{l}\text { Ofrece una explicación en lugar de una } \\
\text { justificación de su punto de vista. } \\
(3 \text { puntos. })\end{array}$ \\
\cline { 2 - 3 } & Repite su punto de vista. & $\begin{array}{l}\text { Repite su punto de vista sin ofrecer } \\
\text { razones. } \\
(2 \text { puntos. })\end{array}$ \\
\cline { 2 - 3 } & Hace comentarios irrelevantes. & $\begin{array}{l}\text { Hace comentarios irrelevantes. } \\
(1 \text { punto. })\end{array}$ \\
\hline No intenta responder. & $\begin{array}{l}\text { No sabe. } \\
(0 \text { puntos. })\end{array}$ \\
\hline
\end{tabular}

En la pregunta 4 (Tabla 4), que evalúa la capacidad argumentativa para contraargumentar, cobra relevancia la dimensión de evaluación contraargumentación. Asimismo, al enfrentarse a una argumentación en contra, esto nos permitió distinguir a los entrevistados que mostraron dudas sobre su punto de vista de aquellos cuya convicción siguió siendo firme, de acuerdo con la dimensión convicción.

En esta pregunta, de manera excepcional, se asignó la misma puntuación a las dos categorías del nivel superior. Esta decisión se tomó porque, como puede verse, no hay ninguna dimensión de evaluación que distinga las dos categorías superiores. Cuando un entrevistado no aceptó la contraargumentación, eso significa que cuestionó las premisas del contraargumento que le presentó el entrevistador. Cuando un entrevistado ofreció razones que rebatían la contraargumentación, eso significa que puso en cuestión la pertinencia del contraargumento para su propio punto de vista. Como explica Marraud (2017), ambas son formas de contraargumentar, que él 
denomina “objeción" y "recusación" respectivamente, y que pueden considerarse argumentativamente equivalentes.

Tabla 4. Dimensiones pregunta 4

\begin{tabular}{|c|c|c|c|c|}
\hline \multicolumn{4}{|c|}{ Dimensiones de evaluación } & Tipo de respuesta \\
\hline \multirow[t]{5}{*}{$\begin{array}{l}\text { Intenta } \\
\text { responder. }\end{array}$} & \multirow[t]{3}{*}{$\begin{array}{l}\text { Responde a la } \\
\text { contraargumentación. }\end{array}$} & \multirow[t]{2}{*}{ No duda. } & \multirow[t]{2}{*}{$\begin{array}{l}\text { Da razones } \\
\text { argumentativamente } \\
\text { pertinentes. }\end{array}$} & $\begin{array}{l}\text { No acepta la } \\
\text { contraargumentación. } \\
\text { (4 puntos.) }\end{array}$ \\
\hline & & & & $\begin{array}{l}\text { Ofrece razones que } \\
\text { rebaten la } \\
\text { contraargumentación. } \\
\text { (4 puntos.) }\end{array}$ \\
\hline & & \multicolumn{2}{|l|}{ Duda. } & $\begin{array}{l}\text { Muestra dudas sobre } \\
\text { su punto de vista. } \\
\text { ( } 3 \text { puntos.) }\end{array}$ \\
\hline & \multirow[t]{2}{*}{$\begin{array}{l}\text { Ignora la } \\
\text { contraargumentación }\end{array}$} & \multicolumn{2}{|c|}{$\begin{array}{l}\text { Da razones argumentativamente } \\
\text { pertinentes. }\end{array}$} & $\begin{array}{l}\text { Ofrece más razones a } \\
\text { favor de su punto de } \\
\text { vista. } \\
\text { (2 puntos.) }\end{array}$ \\
\hline & & \multicolumn{2}{|c|}{ Hace comentarios irrelevantes. } & $\begin{array}{l}\text { Responde con } \\
\text { comentarios evasivos. } \\
\text { (1 punto.) }\end{array}$ \\
\hline \multicolumn{4}{|c|}{ No intenta responder. } & $\begin{array}{l}\text { No sabe. } \\
\text { (0 puntos.) }\end{array}$ \\
\hline
\end{tabular}

Por último, en la pregunta 7 (Tabla 5), en la que se pregunta explícitamente al entrevistado si cree que puede estar equivocado, las dimensiones de evaluación son las mismas que en la pregunta 4, con una excepción: se tomó en consideración también la dimensión explicitud. Esto sirvió para distinguir entre aquellos entrevistados que afirmaron explícitamente que no podían estar equivocados y aquellos que, aunque contraargumentaron, no hicieron esa afirmación explícita. 
Tabla 5. Dimensiones pregunta 7

\begin{tabular}{|c|c|c|c|c|}
\hline \multicolumn{4}{|c|}{ Dimensiones de evaluación } & Tipo de respuesta \\
\hline \multirow[t]{5}{*}{$\begin{array}{l}\text { Intenta } \\
\text { responder. }\end{array}$} & \multirow[t]{3}{*}{$\begin{array}{l}\text { Responde a la } \\
\text { contraargumentación. }\end{array}$} & \multirow[t]{2}{*}{ No duda. } & $\begin{array}{l}\text { Hace explícito su } \\
\text { desacuerdo. }\end{array}$ & $\begin{array}{l}\text { Afirma que no puede estar } \\
\text { equivocado y aporta } \\
\text { razones. } \\
\text { ( } 5 \text { puntos.) }\end{array}$ \\
\hline & & & $\begin{array}{l}\text { Su desacuerdo es } \\
\text { implícito. }\end{array}$ & $\begin{array}{l}\text { No dice explícitamente que } \\
\text { puede estar equivocado y } \\
\text { ofrece razones. } \\
\text { (4 puntos.) }\end{array}$ \\
\hline & & \multicolumn{2}{|l|}{ Duda. } & $\begin{array}{l}\text { Reconoce que puede estar } \\
\text { equivocado. } \\
\text { ( } 3 \text { puntos.) }\end{array}$ \\
\hline & \multirow[t]{2}{*}{$\begin{array}{l}\text { Ignora la } \\
\text { contraargumentación. }\end{array}$} & \multicolumn{2}{|c|}{ No da razones. } & $\begin{array}{l}\text { Afirma que no puede estar } \\
\text { equivocado sin aportar } \\
\text { razones. } \\
\text { ( } 2 \text { puntos.) }\end{array}$ \\
\hline & & \multicolumn{2}{|c|}{ Hace comentarios irrelevantes. } & $\begin{array}{l}\text { Responde con comentarios } \\
\text { evasivos. } \\
\text { (1 punto.) }\end{array}$ \\
\hline \multicolumn{4}{|c|}{ No intenta responder. } & $\begin{array}{l}\text { No sabe. } \\
\text { (0 puntos.) }\end{array}$ \\
\hline
\end{tabular}

El puntaje total en virtud de la suma de cada dimensión es de 15 puntos, siendo este puntaje el máximo de argumentatividad que un hablante natural, de acuerdo con nuestro instrumento, pudo obtener. Así, y en un gradiente, desde 0 a 15 puntos se pudo obtener, como se verá en la próxima sección, las distribuciones de los perfiles de los hablantes según las variables consideradas en este estudio. La puntuación de cada hablante fue otorgada por un proceso de evaluación cualitativa, realizado de manera independiente por quienes firman el presente artículo, siguiendo las definiciones de las categorías indicadas en las Herramientas de análisis y las tablas 2, 3, 4 y 5. Cuando los evaluadores (2) discreparon sustancialmente en sus evaluaciones, se realizó un panel de discusión para dirimir los casos.

\section{Hallazgos Y Discusión}

En primer lugar, daremos cuenta de los hallazgos generales, comparando los resultados obtenidos por adultos mayores y jóvenes adultos. Como se puede apreciar en la Figura 1, los jóvenes adultos, que corresponde a la muestra de estudiantes universitarios, claramente tienen una disposición a argumentar más desarrollada. Es sensible notar que solo los adultos mayores obtuvieron puntuaciones muy bajas (entre 1 a 7 puntos), lo que muestra con nitidez que se debería despejar qué variable específica determina ese declive. 


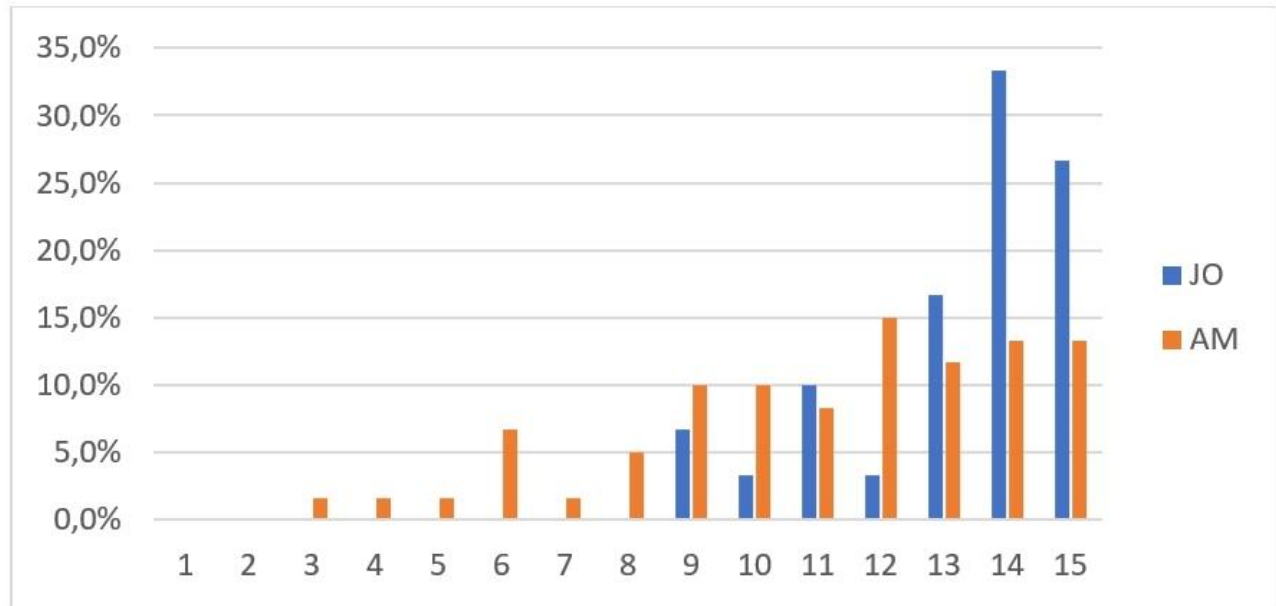

Figura 1. Argumentatividad general en AM y JO

Como se observa en la Figura 1, los JO se concentran mayoritariamente en las puntuaciones más altas (entre 13 a 15), lo que podría deberse a que la muestra está compuesta por estudiantes universitarios, quienes, en los días de la realización de las entrevistas, estaban en sus actividades regulares de clases utilizando la competencia argumentativa. Sin embargo, como se verá luego cuando se comenten desagregadamente los datos de cada pregunta, hay diferencias importantes que comentar dentro del grupo de JO, y también respecto de los AM.

En relación con los puntajes encontrados de acuerdo con la variable edad, la 294 Figura 2 indica que, según las distribuciones etarias propuestas, las puntuaciones altas en argumentatividad se encuentran distribuidas entre JO y AM que están en el rango de edad en que la madurez lingüística, en general, se despliega (Caplan et al., 2011; Dede et al., 2004; Kemper et al., 2001; Kemper y Sumner, 2001; Mackenzie, 2000; Singer et al., 2003; Wingfield y Stine-Morrow, 2000).

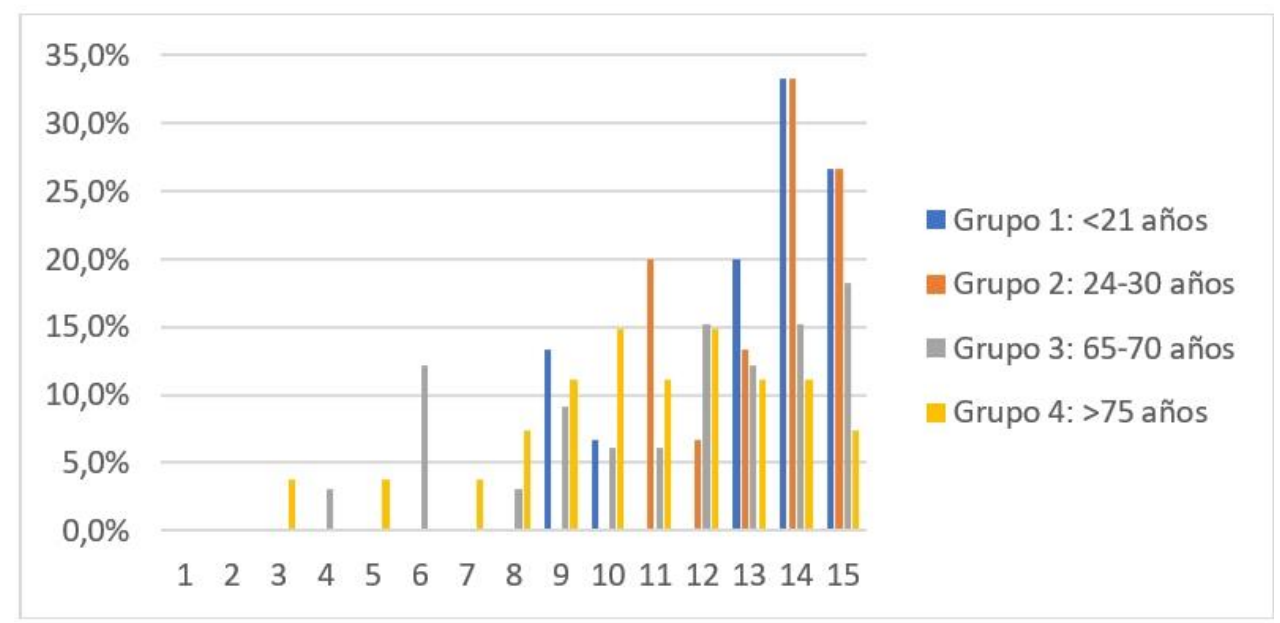

Figura 2. Argumentatividad según tramos etarios en AM y JO

La Figura 2 muestra que, en general, a mayor juventud mayor argumentatividad. Los grupos etarios 1, 2 y 3 tienen, proporcionalmente, mejores puntuaciones. Es interesante notar, sin embargo, que algunos hablantes del grupo 
etario 1 (JO menores a 21) también obtuvieron puntuaciones medias entre 8 y 10 puntos, lo que podría indicar que en este grupo hay diferencias en el ritmo de desarrollo de la capacidad de generar argumentos. Una investigación posterior debería despejar qué factores podrían determinar esas diferencias en el ritmo del desarrollo de la competencia (quizás se vincule con la necesidad de desarrollar otras subcompetencias lingüísticas tales como disponibilidad léxica, memoria semántica, o quizás se vincule a factores de motivación).

Por su parte, en la Figura 3 se presentan los resultados vinculados con la variable género:

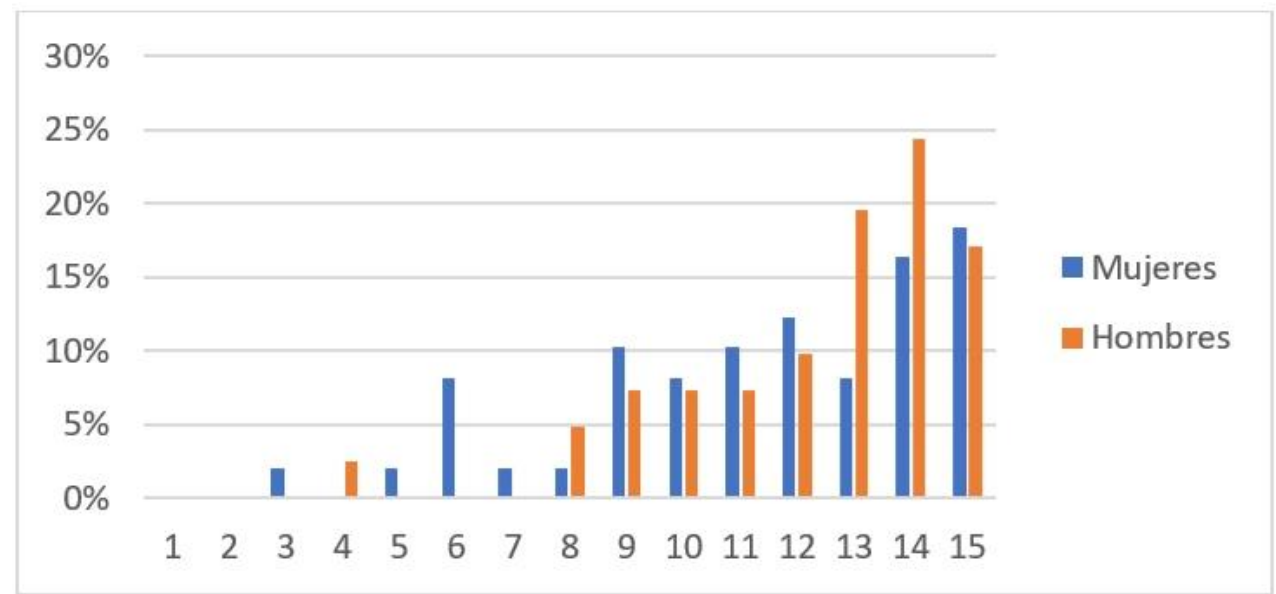

Figura 3. Argumentatividad según género en AM y JO

De acuerdo con los resultados obtenidos, los hombres tuvieron mejores puntuaciones que las mujeres en argumentatividad. Sin embargo, cuando se observan los resultados dentro del grupo de los AM (Figura 4), las mujeres obtienen una cantidad mayor de puntajes máximos $\mathrm{y}$, mirando el grupo de AM en general, entre hombres y mujeres habría un promedio de puntaje similar en argumentatividad. De este modo, la disposición a argumentar estaría distribuida de forma equivalente según género. 


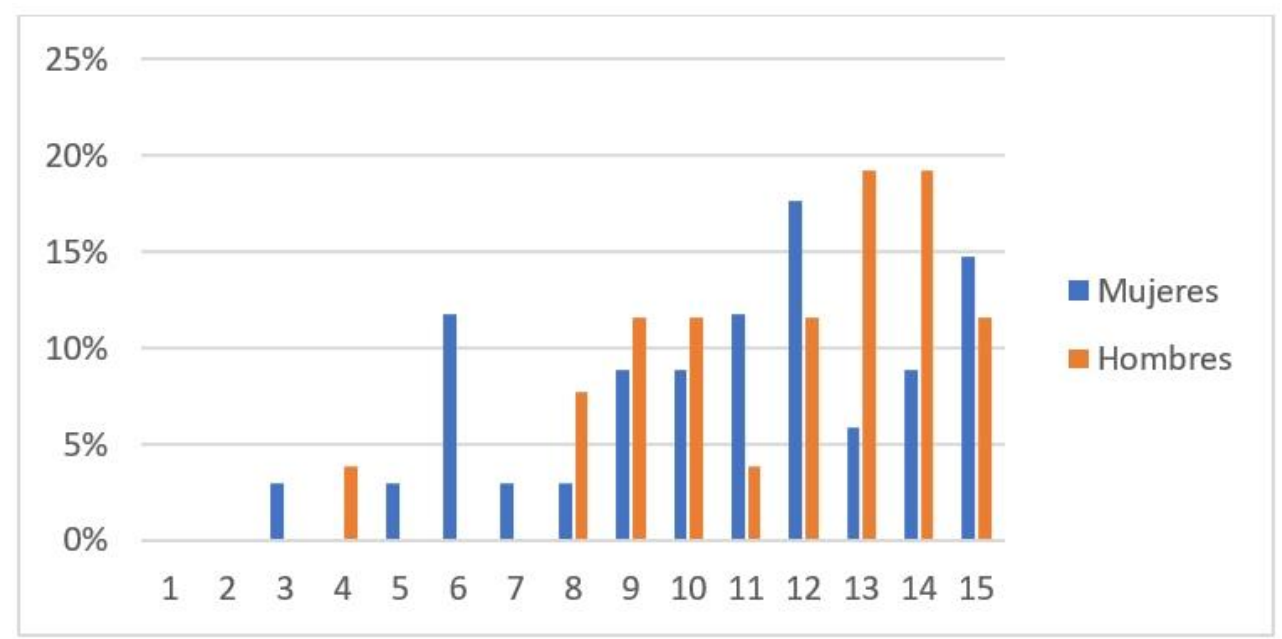

Figura 4. Argumentatividad según género en AM

Ahora bien, los hallazgos específicos se relacionan con los datos provenientes de cada pregunta. Así, en relación con la capacidad de exponer un punto de vista (Figura 5), se obtuvo lo siguiente:

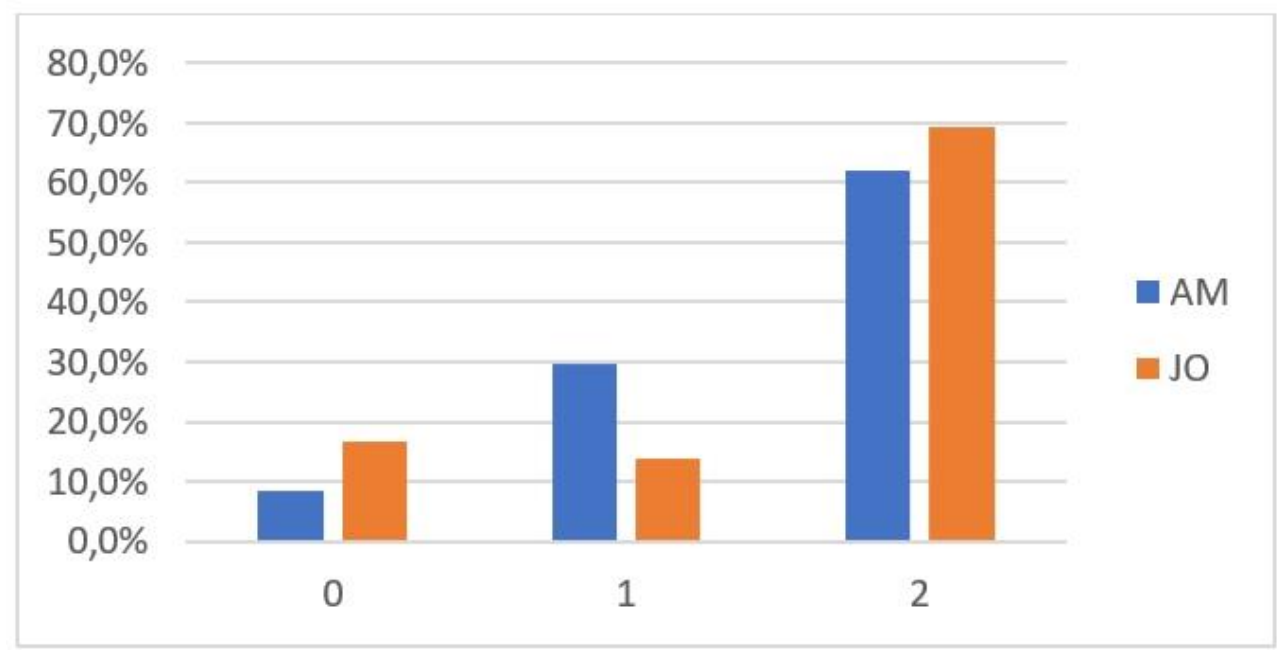

Figura 5. Pregunta 1. Punto de vista en AM y JO

Si bien los jóvenes fueron capaces de presentar un punto de vista con razones en mayor proporción que los adultos mayores, también en mayor proporción no presentaron un punto de vista bien definido, vale decir, fueron más vagos, incompletos o, definitivamente, hay un grupo entre ellos que no entiende la petición de expresar una opinión clara. Estos datos convergen con otras investigaciones realizadas relativas a la vaguedad e incompletud del discurso argumentativo en jóvenes (Felton y Kuhn, 2001; Santibáñez, 2019; Santibáñez et al., 2016).

La Figura 6, correspondiente a la pregunta en la que se les solicitó que dieran más razones para apoyar el punto de vista expresado (pregunta 2 del cuestionario), entrega datos interesantes: 


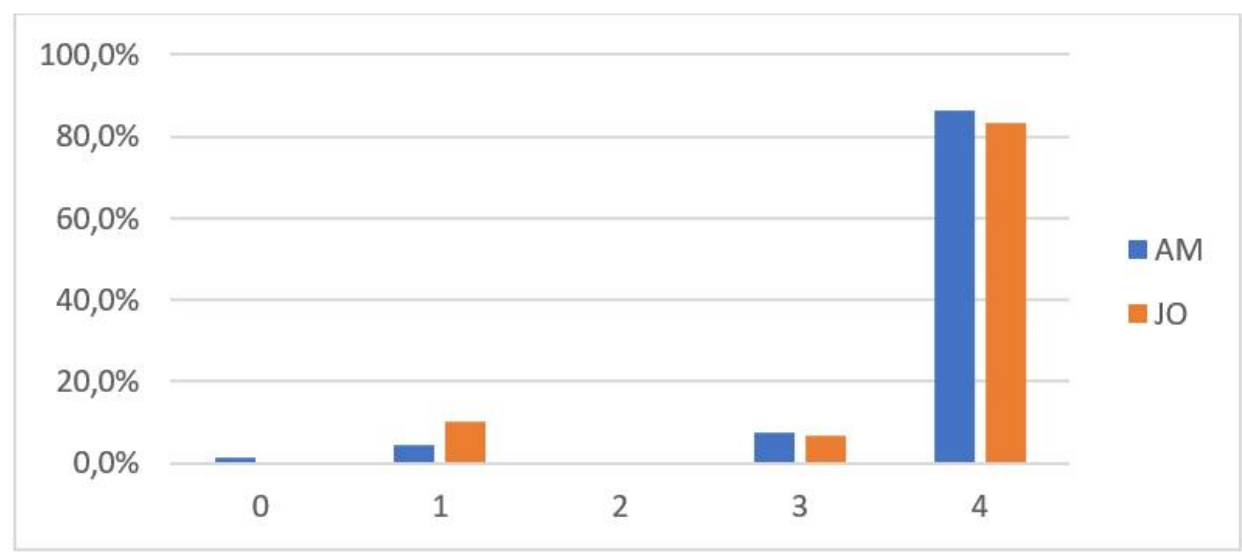

Figura 6. Pregunta 2. Petición de razones en apoyo al punto de vista

Debe recordarse que nuestra tabla evaluativa consideró para la pregunta 2 que una persona obtiene 4 puntos si da razones argumentativamente pertinentes, 3 puntos si explica en lugar de argumentar, 2 puntos si repite su punto de vista sin ofrecer razones, 1 punto si hace comentarios irrelevantes, y 0 si contestó que no sabe. Pues bien, en general se observa una capacidad casi idéntica entre AM y JO de expresar razones en apoyo al punto de vista, y solo algunos casos de JO no fueron capaces en absoluto (obtuvieron 0 puntos). Aunque inicialmente se consideró pertinente añadir una categoría para aquellas respuestas que consistieran solo en una repetición del punto de vista, sin ofrecer razones, en la Figura 6 puede verse que ninguno de los entrevistados respondió la pregunta 2 de esta manera. Este dato es interesante porque muestra que este error argumentativo, que inicialmente consideramos que era probable que nos encontráramos, resulta ser raro o inexistente tanto entre AM como entre JO.

Las preguntas 4 y 7 eran de mayor complejidad, pues involucraron el reconocimiento por parte del entrevistado del uso del esquema de analogía con función contraargumentativa en el comentario del entrevistador (pregunta 4), y la sugerencia en el comentario del entrevistador de la posibilidad de que estuviera en un error, esto es, se le pedía la revisión de su creencia manifestada en su punto de vista. Los resultados en relación con la pregunta 4 se presentan en la Figura 7:

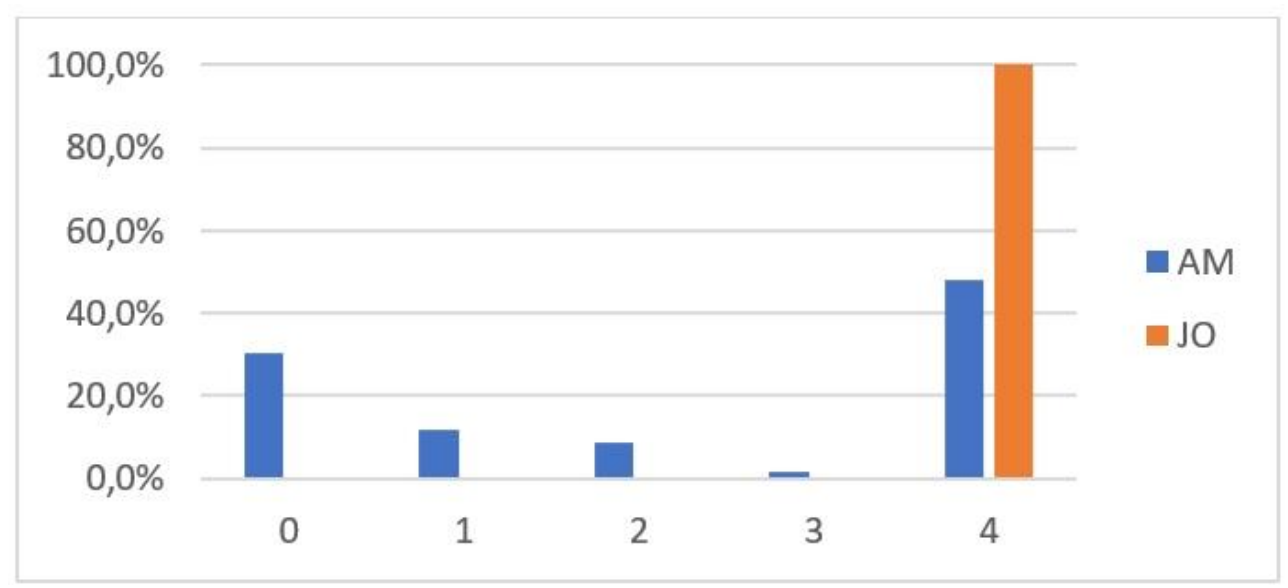

Figura 7. Pregunta 4. Contra argumentación vía analogía 
Obviamente, llama la atención de la Figura 7 que los JO en su totalidad hayan obtenido la puntuación máxima de 4 puntos, que evaluativamente se consideró como aquel agente que responde a la contraargumentación, no duda y da razones argumentativamente pertinentes contra la contraargumentación. Según nuestro análisis, este resultado se debió al hecho de que los entrevistadores del grupo de JO se desviaron ligeramente de la pregunta original, formulándola de tal manera que invitaba a los entrevistados a rebatir la contraargumentación. Prueba de ello es el hecho de que la inmensa mayoría de respuestas corresponden al tipo "Ofrece razones que rebaten la contraargumentación", y no al rechazo de la contraargumentación, que también valía 4 puntos. En consecuencia, debemos concluir que este resultado está sesgado por la manera como se realizó la entrevista. En relación con los resultados de la pregunta 4 en los AM, llama la atención que obtuvieron un porcentaje importante de casos en los que el agente simplemente no respondió porque no supo cómo hacerlo, lo que quizás es indicativo de que contenidos argumentativos que contienen analogías son de una complejidad que no es fácil de procesar. Pero también es relevante indicar que en los AM hubo casos que obtuvieron puntuación 3, que mostraría una capacidad de revisión epistémica, dimensión de la argumentatividad clave para considerar a un agente dispuesto a desarrollar controversias y aceptar sus consecuencias.

En relación con la pregunta 7 del cuestionario suministrado, y que es la última que analizamos, se observa la siguiente información (Figura 8):

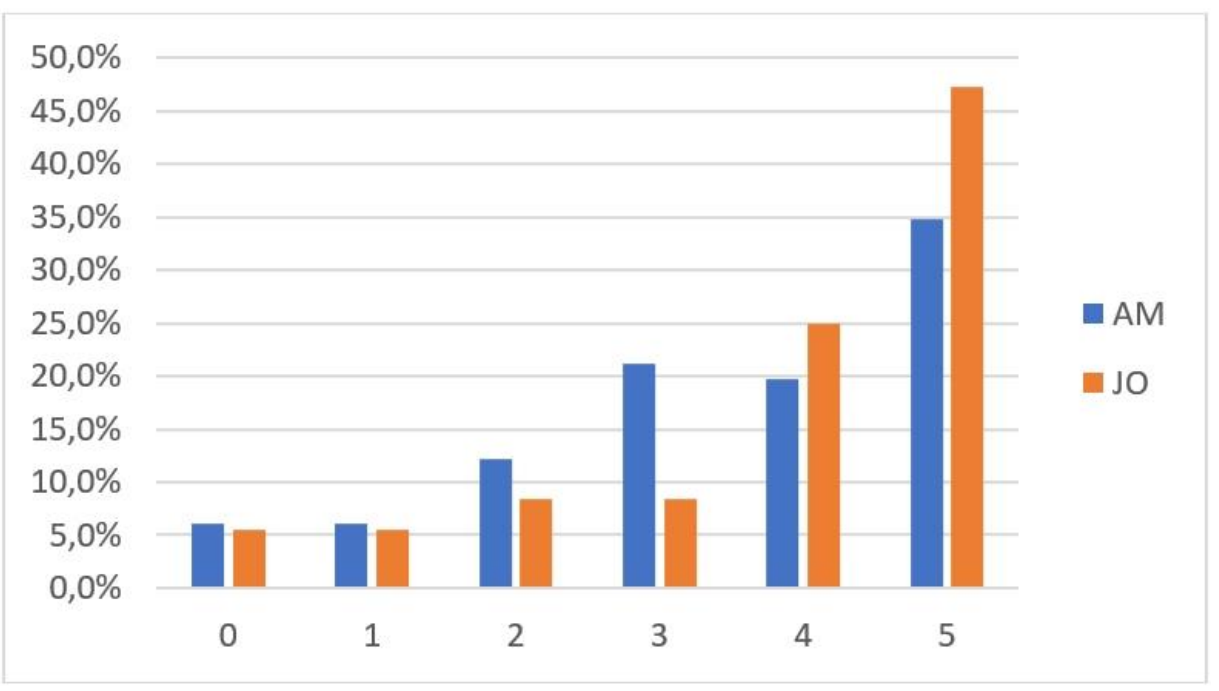

Figura 8. Pregunta 7. Contraargumentación por refutación

Como puede verse, un porcentaje importante de entrevistados (AM y JO) obtuvo las puntuaciones más altas (4 y 5), que en la rúbrica de evaluación cualitativa se registraron como aquel agente que expresó su desacuerdo explícita o implícitamente frente a lo que el entrevistador avanzó. Aunque algunos de estos hablantes no dijeron explícitamente que podían estar equivocados, ofrecieron razones defendiendo su punto de vista inicial. En este gráfico, es relevante el hecho de que es mucho mayor el porcentaje de AM que reconocieron que pudieron estar equivocados que el porcentaje de JO, lo que orienta hacia generalizar que a mayor edad hay una 
mayor capacidad de revisión epistémica, menos dogmatismo. Ambos grupos etarios tuvieron una distribución similar respecto de ignorar la contraargumentación contenida en el comentario de la pregunta 7, que involucró en el discurso de los entrevistados la inexistencia de razones afirmando de todos modos que no podían estar equivocados (no obstante que los AM tuvieron un porcentaje levemente mayor que los JO, con puntuación 2), o afirmando su no equívoco con comentarios simplemente evasivos. También son similares en puntuación 0 , que se relacionó con la conducta de falta de esfuerzo por responder, particularmente porque no sabía cómo hacerlo.

\section{CONCLUSIONES}

La argumentatividad de las personas puede depender, obviamente, de muchos factores, entre ellos, el contexto en el que ocurre una diferencia de opinión, el estado del hablante (cansancio por trabajo, por ejemplo), los vínculos entre quienes participan, el objetivo de la discusión, etc. Reconociendo estas contingencias u otros factores más estructurales, como nivel educativo del hablante (asumiendo que en efecto esta variable juega un rol importante), es no obstante clave hacer ver que la argumentatividad, definida aquí como una predisposición a defender puntos de vista sobre cuestiones controvertidas y a refutar las posturas de otras personas sobre esas cuestiones, se activa más recurrentemente de lo que conscientemente podemos juzgar, y todo esfuerzo (o falta del mismo) por terminar una diferencia de opinión debe verse como una actitud propia de la argumentatividad del hablante.

Lo que nos muestran los resultados, de este modo, es que la edad sí es un factor particular que determina parte de la capacidad/actitud de argumentar. Hemos visto que a mayor edad menos argumentatividad; que el género del hablante, al parecer, no nos dice a priori nada sobre si se acerca o evita la argumentación (aunque las mujeres AM de nuestra muestra tienen más casos de puntuaciones máximas); y que la argumentatividad tendría un ritmo de desarrollo que aún se debe investigar (que se observa a partir del dato de que solo JO menores de 21 años tuvieron puntuaciones relativamente bajas).

Nos parece importante enfatizar un hallazgo específico. Requeridos los entrevistados por proveer más razones (pregunta 2 del cuestionario) en apoyo al punto de vista, los resultados indican que es en el grupo de los AM en el que se aprecia mayor sofisticación de la argumentatividad. Y esto es muy relevante, ya que la exposición de razones, como se ha indicado anteriormente, se puede considerar como el elemento central del comportamiento argumentativo. De forma metafórica, se puede decir que el avance de razones es el corazón de la práctica argumentativa; a través de ella se puede incluso observar el interés que un hablante tiene por sus semejantes con quienes mantiene la controversia. Aquí se podría especular que, en principio, dado que el AM tiene mayor consciencia de los grados de dependencia con los otros, hace un mayor esfuerzo por avanzar razones, frente a un comportamiento 
más juvenil que tiene un perfil más egocéntrico y que se consideraría más autosuficiente.

\section{REFERENCIAS}

Caplan, D., Dede, G., Waters, G., Michaud, J., y Tripodis, Y. (2011). Effects of age, speed of processing, and working memory on comprehension of sentences with relative clauses. Psychology and Aging, 26(2), 439-450. https://doi.org/10.1037/a0021837.

Dede, G., Caplan, D., Kemtes, K., y Waters, G. (2004). The relationship between age, verbal working memory, and language comprehension. Psychology and Aging, 19(4), 601-616. https://doi.org/10.1037/0882-7974.19.4.601.

Dessalles, J.-L. (2007). Why We Talk. The Evolutionary Origins of Language (Trad. J. Grieve). Oxford University Press. (Obra original publicada en 2000 bajo el título de Aux origines du language. Hermès Science Publications).

Evans, J.S.B.T. (2010). Thinking Twice. Two Minds in one brain. Oxford University Press.

Evans, J., y Frankish, K. (Eds.) (2009). In two minds. Dual processes and beyond. https://psycnet.apa.org/doi/10.1093/acprof:oso/9780199230167.001.0001.

Felton, M., y Kuhn, D. (2001). The Development of Argumentative Discourse Skill.

Discourse Processes, 32(2-3),
https://www.tc.columbia.edu/faculty/dk100/faculty-

135-153. profile/files/001_Thedevelopmentofaugumentivediscourseskills.pdf.

Goffman, E. (1959). The Presentation of Self in everyday Life. Overlook Press.

Goffman, E. (1967). Interaction Ritual: Essays in Face-to-Face Behaviour. Aldine.

Goffman, E. (1969). Strategic Interaction. University of Pennsylvania Press.

Hample, D. (2005). Arguing: Exchanging Reasons Face to Face. Lawrence Erlbaum Associates.

Hample, D. (2018). Interpersonal Arguing. Peter Lang.

Hample, D., y Anagondahalli, D. (2015). Understandings of Arguing in India and the United States: Argument Frames, Personalization of Conflict, Argumentativeness, and Verbal Aggressiveness. Journal of Intercultural Communication Research, 44(1), 1-26. https://doi.org/10.1080/17475759.2014.1000939.

Hample, D., y Dallinger, J.M. (7-10 de noviembre de 1985). Cognitive Editing of

Argument Strategies. Presentado en the Annual Meeting of Speech Communication Association, Denver, Colorado, Estados Unidos.

https://files.eric.ed.gov/fulltext/ED266505.pdf.

Hample, D., Richards, A., y Skubisz, C. (2013). Blurting. Communication Monographs, 80(4), 503-532. https://doi.org/10.1080/03637751.2013.830316.

Hample, D., Warner, B., y Young, D. (2009). Framing and Editing Interpersonal Arguments. Argumentation, 23(1), 21-37. https://doi.org/10.1007/s10503-008-9107$\mathrm{x}$. 
Infante, D.A., y Rancer, A.S. (1982). A conceptualization and measure of argumentativeness. Journal of Personality Assessment, 46(1), 72-80. https://doi.org/10.1207/s15327752jpa4601_13.

Infante, D.A., y Rancer, A.S. (1996). Argumentativeness and Verbal Aggression: A Review of Recent Theory and Research. Annals of the International Communication Association, 19(1), 319-351. https://doi.org/10.1080/23808985.1996.11678934.

Infante, D.A., y Wigley, C.J. (1986). Verbal aggressiveness: An interpersonal model and measure. Communication Monographs, 53(1), 61-69. https://doi.org/10.1080/03637758609376126.

Kahneman, D. (2011). Thinking, fast and slow. Allen Lane.

Kemper, S., Greiner, L.H., Marquis, J.G., Prenovost, K., y Mitzner, T.L. (2001). Language decline across the life span: findings from the nun study. Psychology and Aging, 16(2), 227-239.

Kemper, S., y Sumner, A. (2001). The structure of verbal abilities in young an older adults. Psychology and Aging, 16(2), 312-322.

Mackenzie, C. (2000). Adult spoken discourse: the influences of age and education. International Journal of Language \& Communication Disorders, 35(2), 269-285. https://doi.org/10.1080/136828200247188.

Manktelow, K., Over, D., y Elqayam, S. (Eds.). (2011). The Science of Reason. A Festschrift for Jonathan St. B.T. Evans. Psychology Press.

Marraud, H. (2017). De las siete maneras de contraargumentar. Quadripartita Ratio, (4), 52-57.

http://www.quadripartitaratio.cucsh.udg.mx/index.php/QR/article/view/40.

Mercier, H. (2011a). On the Universality of Argumentative Reasoning. Journal of Cognition and Culture, 11(1-2), 85-113. https://doi.org/10.1163/156853711X568707.

Mercier, H. (2011b). Reasoning Serves Argumentation in Children. Cognitive Development, 26(3), 177-191. https://doi.org/10.1016/j.cogdev.2010.12.001.

Mercier, H. (2011c). What good is moral reasoning? Mind E Society, 10(2), 131-148. https://doi.org/10.1007/s11299-011-0085-6.

Mercier, H. (2011d). When Experts Argue: Explaining the Best and the Worst of Reasoning. Argumentation, 25(3), 313-327. https://doi.org/10.1007/s10503-0119222-y.

Mercier, H. (2012). Looking for Arguments. Argumentation, 26(3), 305-324. https://doi.org/10.1007/s10503-011-9256-1.

Mercier, H. (2020). Not Born Yesterday. The Science of Who We Trust and What We Believe. Princeton University Press.

Mercier, H., y Landemore, H. (2012). Reasoning Is for Arguing: Understanding the Successes and Failures of Deliberation. Political Psychology, 33(2), 243-258. https://doi.org/10.1111/j.1467-9221.2012.00873.x.

Mercier, H., y Sperber, D. (2009). Intuitive and reflective inferences. En J.S.B.T. Evans y K. Frankish (Eds.), In two minds: Dual processes and beyond (pp. 149-170). https://doi.org/10.1093/acprof:oso/9780199230167.003.0007. 
Mercier, H., y Sperber, D. (2017). The Enigma of Reason. A New Theory of Human Understanding. Allen Lane.

Paglieri, F., y Castelfranchi, C. (2010). Why argue? Towards a costs-benefit analysis of argumentation. Argument $\mathcal{E}$ Computation, 1(1), 71-91. https://doi.org/10.1080/19462160903494584.

Rancer, A.S., y Avtgis, T.A. (2006). Argumentative and Aggressive Communication: Theory, Research, and Application. http://dx.doi.org/10.4135/9781452225722.

Rancer, A.S., y Infante, D.A. (1985). Relations between motivation to argue and the argumentativeness of adversaries. Communication Quarterly, 33(3), 209-218. https://doi.org/10.1080/01463378509369599.

Santibáñez, C. (2014). ¿Para qué sirve argumentar? Problematizando teórica y empíricamente el valor y la función de la argumentación. Círculo de Lingüística Aplicada la la Comunicación, 58, 163-205. https://doi.org/10.5209/rev_CLAC.2014.v58.45474.

Santibáñez, C. (2019). Generosos y empáticos o ¿pragmáticos y utilitaristas?: los argumentos del adulto mayor chileno frente a un dilema social. Lingüística y Literatura, 40(75), 174-208. https://doi.org/10.17533/udea.lyl.n75a09.

Santibáñez, C., y Hample, D. (2015). Orientations toward interpersonal arguing in Chile. Pragmatics, 23(3), 453-476. http://dx.doi.org/10.1075/prag.25.3.06san.

Santibáñez, C., Migdalek, M., y Rosemberg, C. (2016). Estilos argumentativos en jóvenes universitarios chilenos: Implicancias para una pedagogía colectiva. En C. Santibáñez (Comp.), Ecología Argumentativa Universitaria: desde la realidad a los conceptos (pp. 161-186). Concepción: Editorial Cosmigonon.

Singer, T., Verhaeghen, P., Ghisletta, P., Lindenberger, U., y Baltes, P.B. (2003). The Fate of cognition in very old age: six-years longitudinal findings in the Berlin Aging Study (BASE). Psyhology and Aging, 18(2), 318-331. https://doi.org/10.1037/0882-7974.18.2.318.

Sperber, D. (2001). An Evolutionary Perspective on Testimony and Argumentation. Philosophical Topics, 29(1-2), 401-413.

Sperber, D., Clément, F., Heintz, C., Mascaro, O., Mercier, H., Origgi, G., y Wilson, D. (2010). Epistemic Vigilance. Mind and Language, 25(4), 359-393. https://doi.org/10.1111/j.1468-0017.2010.01394.x.

Sperber, D., y Mercier, H. (2012). Reasoning as a Social Competence. En H. Landemore y J. Elster (Eds.), Collective Wisdom: Principles and Mechanisms (pp. 368-392). Cambridge University Press.

Stanovich, K.E. (2010). Rationality and Reflective Mind. https://doi.org/10.1093/acprof:oso/9780195341140.001.0001.

Stanovich, K.E., y Toplak, M.E. (2012). Defining features versus incidental correlates of Type 1 and Type 2 processing. Mind and Society, 11(1), 3-13. https://doi.org/10.1007/s11299-011-0093-6.

Wingfield, A., y Stine-Morrow, E.A.L. (2000). Language and Speech. En F.I.M. Craik y T.A. Salthouse (Eds.), The Handbook of Aging and Cognition (2a ed.) (pp. 359-416). Lawrence Erlbaum Associates. 